\title{
Effectiveness of a Cognitive Behavioral Therapy-Based Exercise Program for Healthy Employees
}

\author{
Sayuri Takechi1, Toshiaki Kikuchi2, Shiori Horisawa3 ${ }^{3}$, Atsuo Nakagawa4, \\ Kimio Yoshimura5, Masaru Mimura1 \\ ${ }^{1}$ Department of Neuropsychiatry, Keio University School of Medicine, Tokyo, Japan \\ ${ }^{2}$ Department of Neuropsychiatry, Kyorin University School of Medicine, Tokyo, Japan \\ ${ }^{3}$ Institute for Integrated Sports Medicine, Keio University School of Medicine, Tokyo, Japan \\ ${ }^{4}$ Center for Clinical Research, Keio University School of Medicine, Tokyo, Japan \\ ${ }^{5}$ Department of Health Policy and Management, Keio University School of Medicine, Tokyo, Japan \\ Email: sayuritakechi@keio.jp
}

Received 29 September 2015; accepted 27 November 2015; published 30 November 2015

Copyright (C) 2015 by authors and Scientific Research Publishing Inc.

This work is licensed under the Creative Commons Attribution International License (CC BY). http://creativecommons.org/licenses/by/4.0/ (c) (i) Open Access

\section{Abstract}

Psychological distress has a negative impact on employee health and work performance. However, there is a paucity of studies examining the effects of exercise on the psychological well-being of workers in the field of occupational health. In this study, we evaluated the benefits of a cognitive behavioral therapy (CBT)-based exercise program in reducing psychological distress among employees. A 10-week single-group study was conducted with employees aged 20 to 65 years who had no present or past serious medical illness. Eighty-one subjects participated in a 30-minute health education class instructed by a health-exercise teacher and psychologist, and subsequently completed a 10-week CBT-based exercise program developed by the authors. Post-intervention improvement of psychological distress on the Kessler psychological distress scale (K6) was the primary outcome. Forty-four subjects (54.3\%) completed the program. For the subjects who completed the program, the mean total score on K6 significantly reduced from 4.39 to 3.53, with a mean change of $0.86(p=0.044)$. In addition, the total amount of weekly physical exercise significantly increased $(p=0.043)$ and daily amount of time spent sitting significantly decreased $(p=$ 0.023). These findings suggest that a CBT-based exercise program may reduce the level of psychological distress and increase the amount of daily physical activity of healthy employees. In addition, self-help CBT-based exercise programs may have the potential to cause both positive mental and physical effects among non-clinical samples. Future studies including a control group and with higher completion rate among participants should be performed. 
Keywords

Cognitive Behavioral Therapy, Employee, Exercise, Mental Health, Psychological Distress

\section{Introduction}

Psychological distress is a general phrase used to describe unpleasant feelings or emotions such as sadness, anxiety, anger, or frustration (Pratt, 2009). Psychological distress has a negative impact on daily living and affects the course and prognosis of illnesses such as heart disease and diabetes (Weissman et al., 2015). In recent years, it has become clear that psychological distress is even associated with death (Pratt, 2009). Kilkkinen et al. (2007) reported that $31.3 \%$ of a rural population in Australia experienced moderate to severe psychological distress. In particular, workers tend to experience psychological distress, and in Japan, it has been reported that up to 60\% of employees experience severe psychological distress associated with work. Moreover, the number of workers experiencing psychological distress has risen in the past five years, with larger workloads being identified as one of the causes for this increase (Ministry of Health, Labour, and Welfare, 2013). Using data from a survey of time use and leisure activities, Kuroda (2013) reported that the amount of labor per day during weekdays increased from 8.31 hours to 8.67 hours over the past 10 years. Introduction of a five-day work week system has been cited as a possible cause for this increase (Kuroda, 2010). Severe stress affects cognitive functions, such as attention and memory, and lowers work performance (Yerkes \& Dodson, 1908). Therefore, it is important for the field of occupational health to support psychological distress reduction techniques for employees.

Psychological distress can be reduced through mindfulness and cognitive behavioral therapy (CBT) (Mori et al., 2014; Wolever et al., 2012). Exercise also mitigates psychological distress (Cooney et al., 2013). However, although the impact of exercise on mental health has been suggested, current exercise research targeting employees has primarily examined its effects on physical health (Christensen et al., 2011; Proper et al., 2003). To the best of our knowledge, very few studies have examined the association between exercise and mental health in the field of occupational health. One such study, by Merrill et al. (2011), reported that increasing the activity level of subjects who were obese prior to an intervention for the promotion of health improved mental state indexes such as emotional state, sense of well-being, stress coping, and satisfaction with work. In addition, Herman et al. (2006) reported that when employees exercised, individual health risk status improved and their level of life dissatisfaction decreased. However, in these reports each item was evaluated separately with a unique question, not from multiple points of view using a standardized evaluation scale.

In previous exercise research targeting workers, the results varied depending on the content of the intervention and the location. Moreover, the rate of adherence varied widely, from $40 \%$ to $90 \%$ (Atlantis et al., 2006; Grande, Silva, \& Parra, 2014). The rate of adherence for health promotion programs promoted by the workplace is lower than 50\%, according to Robroek et al. (2009). Thus, proper support is needed to maintain motivation and improve the adherence rate. Support for maintaining motivation is similar to weight loss support for patients with diabetes. CBT, which has been confirmed to be effective for the treatment of depression, has been used to treat patients with various conditions other than mental illnesses in recent years. CBT is based on the premise that dysfunctional attitudes or beliefs are hijacked by maladaptive information processing that leads to psychological and somatic symptoms, and that our thoughts and feelings play a fundamental role in behavior. This "cognitive behavioral model" posits that when dysfunctional thoughts or behaviors are corrected, both acute stress and the risk for subsequent symptoms will be reduced (Beck, 2008; DeRubeis, Siegle, \& Hollon, 2008). CBT programs for chronic pain (Ehde, Dillworth, \& Tuner, 2014), dizziness (Cima et al., 2014), and dieting have been developed (Beck, 2007); Tsiros et al. (2008) verified the effectiveness of a CBT-based program for diet (Tsiros, 2008). We have developed a CBT-based exercise program based on the "The beck diet solution" (Beck, 2007; Beck, 2009), which utilizes skills such as psychological education, mood monitoring, and cognitive reconstruction. In this study, we assessed whether a program involving self-help exercise based on CBT could reduce psychological distress in employees.

\section{Methods}

\subsection{Participants}

Our study was conducted between November 2013 and February 2014 with employees from three offices of a 
general business company. We recruited employees who voluntarily participated in group health guidance at a manufacturing company, and those who met the inclusion criteria were enrolled in the study. The mean length of the participants' workday was approximately 8 hours, which is average in Japan. Regarding labor type, 40\% of participants engaged in manufacturing operations, and $60 \%$ engaged in deskwork.

The subjects were included in the study if they were healthy, between 20 and 65 years old, had no serious diseases or significant medical history, agreed to participate in the study, and were able to exercise. We excluded individuals who were unable to give written consent; who were determined by a physician to be unable to exercise due to conditions such as heart disease, cerebrovascular disease, or orthopedic disease; who had already performed sufficient strength training; or who could not read. The study was approved by the Ethics Committee of the Keio University School of Medicine and was registered within the UMIN Clinical Trials Registry (UMIN000012277).

\subsection{Intervention}

First, subjects were educated about how regular exercise promotes health and prevents diseases such as type 2 diabetes, hypertension, and obesity-related disorders. Supplemental education regarding the growing interest in delineating the role of physical activity and exercise behavior in enhancing wellness was also provided. Afterward, a health-exercise instructor gave participants practical guidance on a specific exercise program. Next, an explanation of dysfunctional thinking or behavior that could prevent engaging in exercise was delivered by a psychologist. Typical thoughts such as "I'm too busy to exercise" and "Not exercising for one day won't make a difference" were discussed, and a specific method to alter such thoughts was offered: cognitive reconstruction. In addition, a mood-monitoring method was explained using a worksheet. The way to perform mood monitoring was as follows: the subjects rated the degree of enjoyment associated with exercise. A self-report scale of 0 to 10 was used $(0=$ no enjoyment, $10=$ greatest sense of enjoyment). Subjects were then provided with a CBT-based exercise program workbook structured as follows: identification and modification of dysfunctional thoughts (i.e., a cognitive reconstruction worksheet), a problem-solving worksheet listing actual problems that prevent exercise, illustrations to demonstrate specific warm-up and cool-down procedures, a recommended exercise routine, and a worksheet to monitor behaviors and accompanying mood changes. The program was designed by ST, AN, KY, and TK in reference to "The Beck diet solution" as described by Beck (Beck, 2007; Beck, 2009). AN and TK, who are CBT experts, and KY, who is a behavioral medicine expert, reviewed the validity of the program design.

Subjects engaged in a 10-week exercise program at home following the workbook. Exercises consisted of a warm-up period, five types of core strength training (squats, lunges, sit-ups, crunches, and arm/leg raises), and a cool-down period. The frequency of exercise was recommended as three days per week. As for the intensity of exercise, we used the concept of "Niko Niko Pace", which refers to the intensity of exercise at which one can maintain a smile, as a guideline for safety and making it easier to form an exercise habit (Higaki, 2013; Tanaka, 2015). Subjects were instructed to aim for an intensity of 11 to 13 on the Borg Rating of Perceived Exertion scale (Borg scale), which is roughly equivalent to the intensity of "Niko Niko Pace". The Borg scale is a measure of physical activity intensity level. Perceived exertion (i.e., how hard participants felt their body was working) is based on the physical sensations a person experiences during physical activity, including increased heart rate, increased respiration or breathing rate, increased sweating, and muscle fatigue (Borg, 1998). On days when a subject exercised, they were instructed to record the type of exercise, the degree of intensity based on the Borg scale, perform mood monitoring before and after exercise, and enter comments after exercise on the monitoring sheet.

\subsection{Evaluation}

We performed an evaluation during a one-week period from the point of group guidance to the beginning of the program - this period was defined as the pre-intervention — and a post-intervention evaluation was performed 10 weeks after the program began. The primary outcome was the participant's psychological distress; secondary outcomes included subjective well-being, mental state including self-esteem, perception of exercise, amount of physical activity performed in one week, work performance, living conditions and lifestyle habits, and body composition values such as weight and abdominal circumference.

The Kessler psychological distress scale (K6) (Furukawa et al., 2002; Kessler et al., 2002) was used as an in- 
dex to evaluate psychological distress; the World Health Organization Subjective Well-being Inventory (WHO SUBI) (Fujinami, Sonoda, \& Ono, 1996; Ono \& Yoshimura, 2008; Sell \& Nagpal, 1992), for subjective wellbeing; and the self-esteem scale (SES) (Mimura \& Griffiiths, 2007; Rosenberg, 1965), for self-esteem. The K6 is a self-administered questionnaire that measures psychological distress and determines an individual's tendencies toward depression and anxiety. The answers to six questions are provided on a five-point scale, with total scores ranging from 0 to 30 . A higher score is associated with a more severe burden of psychological distress. The WHO SUBI, which was developed to measure subjective well-being, is able to separate positive and negative feelings. It consists of 40 questions, and answers are given on a three-point scale. The SES is an evaluation scale for self-esteem that consists of 10 questions, with answers given on a four-point scale.

The amount of physical activity for one week was evaluated using the International Physical Activity Questionnaire (IPAQ) (Craig et al., 2003; Murase et al., 2002). The amount of physical activity was evaluated by intensity (vigorous, moderate, or walking). The answers to the questions required participants to record the time they devoted to each physical activity. In addition, the overall physical activity was calculated by intensity and time, which allowed for assignment of metabolic equivalent (MET) values to the activities: 8 MET for vigorous activity, 4 MET for moderate activity, and 3.3 MET for walking (Craig et al., 2003). Perception of exercise was evaluated by three questions: (1) "How do you feel about exercise (moving the body in general, such as walking and calisthenics)?” (2) "How much actual benefit from exercise on your physical health do you feel?” and (3) "How much actual benefit from exercise on your mental health do you feel?" These questions were answered using a five-point scale. A higher score was associated with a more positive recognition for exercise. Subjective work performance was evaluated on a ten-point scale based on the following statements extracted from the WHO Health and Work Performance Questionnaire (HPQ) (Kessler et al., 2003; Yoshimura et al., 2013): "subjective evaluation of one's own work compared to others," "subjective evaluation of one's work in the last one to two years," and "subjective evaluation of one's own work for the past four weeks". Regarding lifestyle habits, the subjects reported the amount of alcohol and tobacco used, how many times per week breakfast was eaten, how many hours of sleep they got on weekdays and holidays, and the average number of hours worked per day. The amount of alcohol ingested was calculated by assigning $20 \mathrm{~g}$ of alcohol for $500 \mathrm{ml}$ of beer, $180 \mathrm{ml}$ of Japanese sake, $200 \mathrm{ml}$ of wine, or $60 \mathrm{ml}$ of whiskey. In addition, indexes of body composition, weight, body fat, and abdominal circumference were measured using Inbody 470 (InBody Japan Inc., Tokyo, Japan). As a qualitative evaluation after the intervention, we requested that subjects report any adverse events, evaluate their perception of the impact of the exercise on a five-point scale, evaluate their satisfaction with the program on a three-point scale, and note the reasons for their ratings.

\subsection{Analysis}

Statistical analyses were performed using IBM SPSS Statistics 22 for Windows (IBM Corp., Armonk, NY, USA). To analyze the change between pre- and post-intervention assessments, the paired t-test was used for parametric data or McNemar's test was used for categorical variables. Two-tailed $p<0.05$ was considered statistically significant.

\section{Results}

Approximately 240 people received group guidance prior to the intervention. We determined the final sample size based on the status of health education at the company, with 81 people consenting to participate in the study. Of the 81 subjects, 44 (54.3\%) provided useful responses that were used for the analyses during the post-intervention period. The results are shown in Table 1.

Subjects ranged from 31 to 65 years old, with a mean age of $49.4 \pm 11.2$ years. The mean number of years working for the company was 26.1, and many had a stable, long-term job. Most households included a husband or a wife and other persons living in the household; $11.4 \%$ of participants were categorized as single. The mean K6 score was 4.39, and including the SES and SUBI, the overall mental state was relatively stable. However, only 17 (38.6\%) subjects who met the criterion of $1380 \mathrm{METS} / \mathrm{min}$ per week were proposed to have a healthy amount of activity in daily life by the Ministry of Health, Labour, and Welfare, indicating that the amount of exercise was low.

Psychological distress significantly decreased after the intervention, with the pre-intervention K6 score of $4.39 \pm 3.70$ reducing to $3.53 \pm 2.72$ post-intervention $(p=0.044)$. However, the WHO SUBI and SES scores did 
Table 1. Comparison of parameters pre- and post-intervention.

\begin{tabular}{|c|c|c|c|c|}
\hline & $\mathrm{n}$ & Pretest & Posttest & $p$-value \\
\hline Age, y & 44 & $51.3(11.0)$ & - & \\
\hline Sex, male, n (\%) & 44 & $31(70.5)$ & - & \\
\hline Household, living alone, n (\%) & 44 & $5(11.4)$ & - & \\
\hline \multicolumn{5}{|l|}{ Body composition } \\
\hline Body weight, kg & 42 & $65.1(10.8)$ & $65.2(10.4)$ & 0.358 \\
\hline Body fat, kg & 42 & $15.4(5.15)$ & $15.6(5.76)$ & 0.954 \\
\hline Waist, cm & 39 & $79.7(8.26)$ & $79.8(7.90)$ & 0.969 \\
\hline \multicolumn{5}{|l|}{ Life style } \\
\hline Current smoker, n (\%) & 44 & $1(2.3)$ & $0(0.0)$ & - \\
\hline Alcohol intake per week, ml & 43 & $143.4(162.1)$ & $110.0(135.0)$ & 0.075 \\
\hline Days of having breakfast in a week & 42 & $6.23(1.71)$ & $6.60(0.83)$ & 0.104 \\
\hline Sleeping hours on a weekday & 44 & $6.19(0.81)$ & $6.16(0.82)$ & 0.722 \\
\hline Sleeping hours on a holiday & 44 & $7.03(0.95)$ & $7.23(0.91)$ & 0.114 \\
\hline \multicolumn{5}{|l|}{ Physical activity } \\
\hline Vigorous activity per day, h & 44 & $0.14(0.29)$ & $0.17(0.30)$ & 0.265 \\
\hline Moderate activity per day, $\mathrm{h}$ & 44 & $0.12(0.29)$ & $0.11(0.32)$ & 0.717 \\
\hline Walking per day, h & 44 & $0.42(0.54)$ & $0.62(0.76)$ & 0.079 \\
\hline Total physical activity per week, METs-minute & 44 & $1267.9(1302.0)$ & $1612.5(1569.6)$ & $0.043^{*}$ \\
\hline Sitting per day, h & 44 & $6.62(5.39)$ & $4.57(4.04)$ & $0.023 *$ \\
\hline \multicolumn{5}{|l|}{ Perception of exercise } \\
\hline Exercise is fun, score & 43 & $2.93(1.03)$ & $2.95(0.98)$ & 0.822 \\
\hline Exercise is effective for physical fitness, score & 43 & $3.68(0.471)$ & $3.74(0.44)$ & 0.533 \\
\hline Exercise is effective for mental health, score & 43 & $3.52(0.59)$ & $3.44(0.77)$ & 0.456 \\
\hline \multicolumn{5}{|l|}{ Mental health } \\
\hline K6, score & 44 & $4.39(3.70)$ & $3.53(2.72)$ & $0.044 *$ \\
\hline SES, score & 43 & $24.9(2.05)$ & $24.5(2.70)$ & 0.258 \\
\hline SUBI-positive well-being, score & 40 & $37.8(6.78)$ & $39.0(6.91)$ & 0.120 \\
\hline SUBI-negative well-being, score & 40 & $51.3(6.14)$ & $51.7(5.51)$ & 0.206 \\
\hline \multicolumn{5}{|l|}{ Work } \\
\hline Working hours per day & 44 & $8.10(1.94)$ & $8.77(1.36)$ & 0.055 \\
\hline Work performance score & 44 & $6.91(1.83)$ & $6.62(1.62)$ & 0.400 \\
\hline
\end{tabular}

Data are presented as mean (SD), unless mentioned otherwise. K6: The Kessler psychological distress scale. SES: the self-esteem scale. SUBI: the World Health Organization Subjective Well-being Inventory. $P$-value was analyzed for the difference between baseline and endpoint scores, using the paired t-test for parametric data and the chi-square test for nonparametric data. ${ }^{*}$ statistically significant $p$-values $(p<0.05)$.

not show a significant difference pre- and post-intervention. The total amount of physical exercise in one week was $1267.9 \pm 1302.0$ METs-min before the intervention and $1612.5 \pm 1569.6$ METs-min after the intervention, which was a significant increase $(p=0.043)$. The amount of time spent sitting during a day was $6.62 \pm 5.39$ 
hours prior to the intervention and $4.57 \pm 4.04$ hours after the intervention, representing a significant decrease ( $p$ $=0.023$ ).

Perception of exercise, work performance, lifestyle habits, and body composition variables did not show significant differences, although the amount of alcohol ingested showed a decreasing trend (baseline: $143.4 \mathrm{~g} /$ week; endpoint: $110.0 \mathrm{~g} /$ week, $p=0.075$ ) and the mean work hours per day showed an increasing trend (pre: $8.10 \mathrm{~h}$; post: $8.77 \mathrm{~h}, p=0.079$ ).

Adverse events due to the intervention occurred in 12 subjects (27.3\%): muscle pain in 7 (15.9\%), knee pain in 2 (4.5\%), lower back pain in 1 (2.3\%), ankle pain in $1(2.3 \%)$, and weight gain in 1 (2.3\%). No participants had a serious condition. It is noteworthy that there were no differences in age $(p=0.19)$, sex $(\mathrm{p}=0.30)$, or K6 baseline score $(p=0.69)$ between the completion group $(n=45)$ and non-completion group $(n=34)$.

After the intervention, regarding satisfaction with the program, 28 subjects (64\%) reported that the program positively affected their health (responses of "somewhat effective" and "felt positive effects") (Table 2). The breakdown of perceptions about exercise were as follows: regarding the mental impact, the program provided a good change of pace for 16 subjects (36.7\%), stress release for 10 subjects (22.7\%), and reduction in depression and anxiety for 3 subjects (6.8\%); regarding the physical impacts, muscle increases were perceived by 12 participants (27.3\%), improved physical fitness by 10 subjects (22.7\%), reduced body fat by 4 subjects (9.1\%), and decreased pain by 2 subjects (4.5\%). Regarding the degree of satisfaction with the program, 32 subjects (73\%) answered that they were glad they had participated. The reasons for satisfaction included realization of the impacts of exercise (e.g., "increased physical fitness”, “a good change of pace”, and "change in body shape”); descriptions of increases in activity (e.g., "exercise is becoming a habit" and "consciously made it a lifestyle habit”); descriptions of behavioral changes (e.g., "it led to regular visits to the gym” and “instead of drinking after dinner, exercise helped me fall asleep”); and descriptions of the changes in the way subjects recognized exercise (e.g., "I understand the importance of exercise" and "I gained a habit to exercise even if I only have a little time for it”).

\section{Discussion}

The results indicate that a 10-week exercise program based on CBT elements mitigated the psychological distress of healthy employees and increased the amount of physical activity in their daily lives. Although the difference was not significant, there were trends toward decreased alcohol ingestion and increased work hours. This indicates that a CBT-based exercise program has the potential for positive mental and physical effects.

In this study, dysfunctional thoughts or behaviors that prevented exercise adherence were corrected, leading to daily exercise. In addition, effects of monitoring on promoting achievement of goals and behavioral changes have been reported (Harkin et al., 2015). By incorporating self-monitoring materials, exercise adherence was promoted in our program, which led to increased physical activity. In addition, in regards to possible mechanisms between physical activity and enhanced psychological function, Fox (2000) has suggested exercise potentially leads to changes in physical self-worth and self-esteem due to mastering a new task and a greater sense of personal control. Accordingly, in this study, physical self-worth or self-esteem of the subjects increased after they learned a new task that they had not practiced before: exercise. In addition, as the subjects were distanced from the stresses of everyday life, it likely led to lowered psychological distress.

Edries, Jelsma, \& Maart (2013) created an employee wellness program based on CBT and reported that the health-related quality of life and activity level of workers improved. This program was not a CBT-based approach that focused on exercise therapy, but was centered on goal setting, pacing, and self-reflection that aimed to promote self-efficacy. Our program focused on cognitive reconstruction, which is considered a barrier to implementing exercise, and behavioral activation characterized by monitoring activities and mood, which promotes motivation to change behavior and to exercise more. As evidenced by the answers to our open-ended questions, behavioral changes and reconstruction of maladaptive thoughts occurred, and our program demonstrated potential effectiveness in inspiring exercise and increased activity.

This study did not find changes in the SUBI, an index of subjective well-being, or the SES, an evaluation of self-esteem. The reasons for these findings likely involve the small number of subjects, short duration of the program, lack of observation when the program was implemented, and the ceiling effect, as the subjects were healthy individuals. In addition, while the rating scale of the K6 assesses internal psychological symptoms, the SUBI questionnaire is mainly composed of evaluations of the self in social relationships and the SES evaluates 
Table 2. Degree of subjective efficacy and satisfaction of the program.

n

Degree of subjective efficacy considering mental and physical health

Definite
Somewhat
Slightly
Little
Not at all

\section{$\%$}

$10 \quad 22.7$

$18 \quad 40.9$

613.6

$5 \quad 11.4$

$5 \quad 11.4$

Satisfaction with the program

Totally satisfied

Somewhat satisfied
32

9

3
72.7

20.5

6.8

self-esteem based on knowledge of the self. As such, the main factors assessed are different for each scale, and it is possible that the SUBI and SES scores would improve over a period longer than 10 weeks given self-esteem and social cognition are thought to alter after psychological symptoms improve.

In addition, changes in weight, body fat, and abdominal circumference were not found. The period of intervention in this study was limited (10 weeks), but continuing the exercise for a longer period may lead to physical impacts. Alternatively, the amelioration of psychological distress may occur from changes in the body.

Importantly, the amount of physical activity in the subjects' daily lives increased with the implementation of this program. Concomitantly, the amount of time spent sitting was reduced. Answers to the open-ended questions after the intervention included the following: "I was able to understand the importance of exercise", "I realized I have been making excuses for not exercising, pretending I am busy", and "I reconfirmed that health is important to me”. In other words, maladaptive thoughts around exercise were modified, and increased opportunities for self-observation that led to meta-cognitive observation were recognized. We believe these observations occurred because the approach was designed on a CBT foundation. In addition, several subjects noted that after completing the program, they joined athletic gyms or tennis circles; thus, it promoted changes in behavior.

In this study, 54.3\% of subjects provided useful answers. One of the reasons for this low rate is that the post-intervention measurements were only performed on two weekdays because of the characteristics of the participating company and the research costs. Thus, some participants could not attend sessions due to their work schedule. The other reason is that the program was designed to not provide continuous instruction over the course of the research period, but rather one direct intervention for each subject on the day that group health guidance was scheduled. Some instances of insufficient performance could be due to this infrequent instruction. However, among participants who did not provide follow-up data (37 subjects, 45.7\%), the demographic characteristics such as age and gender and the physical/mental factors at baseline were not significantly different from subjects who provided a second response (44 subjects, 54.3\%). In addition, the adherence rate was similar to other interventions in companies (e.g., Robroek et al., 2009), indicating the difficulty of implementing interventions in healthy subjects and in the field of occupational health in general. Robroek \& colleagues (2009) suggest that three elements (incentives, offering a multi-component strategy, and focusing on multiple behaviors rather than physical activity only) increase the level of continued activity. Although our program included the latter two elements, it may benefit from including some type of incentive. In fact, Merrill et al. (2011) suggested that by providing a monetary reward, the participation rate in an exercise program increased by up to $80 \%$. Shortening the interval by increasing the number of interventions in order to have a continuous approach also has the potential to increase the adherence rate (Atlantis et al., 2006; Proper et al., 2003).

Although our CBT-based exercise program was shown to be effective, this research has some limitations. First, the study did not have a control group, so factors could only be compared between pre- and post-intervention assessments; thus, the effectiveness of the exercise program cannot be compared with other interventions. 
To further investigate the effectiveness of this program, a controlled study with a larger number of subjects will be necessary. Second, during the intervention period, monitoring was limited to exercise and mood and other lifestyle aspects were not monitored. Therefore, the confounding effects of environmental factors on the results cannot be excluded. Third, the total amount of physical exercise was evaluated not with recording devices such as a pedometer or an activity monitor, but by writing down daily activity. Therefore, further study is needed to address changes in physical exercise. Fourth, data are missing for some subjects. However, the results did not change when only subjects with complete data were analyzed. Fifth, the time of exercise was not regulated. Some investigators report that the circadian rhythm of the heart rate changes depending on the time of exercise (Carandente et al., 2006), but in the present study, the time of exercise was determined by each individual. Finally, since the subjects of this study were volunteers, they may have already been interested in health and were motivated to exercise. Thus, selection bias cannot be denied. In future studies, the subjects should be randomly assigned to an intervention or control group.

\section{Conclusion}

This study incorporated elements of CBT during a 10-week exercise program that utilized self-help material. We approached dysfunctional thoughts and behaviors against exercise adherence using a "cognitive behavioral model”. As a result, changes in subjects' recognition of exercise and behavior were achieved, leading to increased physical activity on a daily basis. In addition, we were able to confirm that psychological distress decreased with regular exercise. As such, this program was effective in supporting employees' health. In the future, we plan to incorporate measures that improve the rate of adherence and verify the effectiveness of the program via a randomized controlled trial.

\section{References}

Atlantis, E., Chow, C. M., Kirby, A., \& Fiatarone Singh, M. A. (2006). Worksite Intervention Effects on Physical Health: A Randomized Controlled Trial. Health Promotion International, 21, 191-200. http://dx.doi.org/10.1093/heapro/dal012

Beck, A. T. (2008).The Evolution of the Cognitive Model of Depression and Its Neurobiological Correlates. The American Journal of Psychiatry, 165, 969-977. http://dx.doi.org/10.1176/appi.ajp.2008.08050721

Beck, J. S. (2007). The Beck Diet Solution: Train Your Brain to Think Like a Thin Person. Birmingham, Alabama: Oxmoor House.

Beck, J. S. (2009). The Beck Diet Solution (2nd ed.). Birmingham, Alabama: Oxmoor House.

Borg, G. (1998). Borg's Perceived Exertion and Pain Scales. Champaign, Illinois: Human Kinetics.

Carandente, F., Montaruli, A., Roveda, E., Calogiuri, G., Michielon, G., \& La Torre, A. (2006). Morning or Evening Training: Effect on Heart Rate Circadian Rhythm. Sport Sciences for Health, 1, 113-117. http://dx.doi.org/10.1007/s11332-006-0020-0

Christensen, J. R., Faber, A., Ekner, D., Overgaard, K., Holtermann, A., \& Sogaard, K. (2011). Diet, Physical Exercise and Cognitive Behavioral Training as a Combined Workplace Based Intervention to Reduce Body Weight and Increase Physical Capacity in Health Care Workers-A Randomized Controlled Trial. BMC Public Health, 11, 671. http://dx.doi.org/10.1186/1471-2458-11-671

Cima, R. F., Andersson, G., Schmidt, C. J., \& Henry, J. A. (2014). Cognitive-Behavioral Treatments for Tinnitus: A Review of the Literature. Journal of the American Academy of Audiology, 25, 29-61. http://dx.doi.org/10.3766/jaaa.25.1.4

Cooney, G. M., Dwan, K., Greig, C. A., Lawlor, D. A., Rimer, J., Waugh, F. R., McMurdo, M., \& Mead, G. E. (2013). Exercise for Depression. Cochrane Database Systematic Reviews, 9, 1-156.

Craig, C. L., Marshall, A. L., Sjostrom, M., Bauman, A. E., Booth, M. L., Ainsworth, B. E., Pratt, M., Ekelund, U., Yngve, A., Sallis, J. F., \& Oja, P. (2003). International Physical Activity Questionnaire: 12-Country Reliability and Validity. Medicine \& Science in Sports \& Exercise, 35, 1381-1395. http://dx.doi.org/10.1249/01.MSS.0000078924.61453.FB

DeRubeis, R. J., Siegle, G. J., \& Hollon, S. D. (2008). Cognitive Therapy versus Medication for Depression: Treatment Outcomes and Neural Mechanisms. Nature Reviews Neuroscience, 9, 788-796. http://dx.doi.org/10.1038/nrn2345

Edries, N., Jelsma, J., \& Maart, S. (2013). The Impact of an Employee Wellness Programme in Clothing/Textile Manufacturing Companies: A Randomised Controlled Trial. BMC Public Health, 13, 25. http://dx.doi.org/10.1186/1471-2458-13-25

Ehde, D. M., Dillworth, T. M., \& Turner, J. A. (2014). Cognitive-Behavioral Therapy for Individuals with Chronic Pain: Efficacy, Innovations, and Directions for Research. American Psychologist, 69, 153-166. http://dx.doi.org/10.1037/a0035747 
Fox, K. R. (2000). Self-Esteem, Self-Perceptions and Exercise. International Journal of Sport Psychology, 31, $228-240$.

Fujinami, K., Sonoda, A., \& Ono, Y. (1996). Preparation of a Japanese Version of SUBI and Examination of Reliability. The Japanese Journal of Health Psychology, 8, 12-19. (In Japanese)

Furukawa, T., Ono, Y., Uda, H., \& Nakane, M. (2002). Study on a Simple Screening of Mental Illnesses in General Population. Health, Labour and Welfare Science, Special Research Project. (In Japanese)

Grande, A. J., Silva, V., \& Parra, S. A. (2014). Effectiveness of Exercise at Workplace in Physical Fitness: Uncontrolled Randomized Study. Einstein (Sao Paulo), 12, 55-60. http://dx.doi.org/10.1590/S1679-45082014AO2956

Harkin, B., Webb, T. L., Chang, B. P., Prestwich, A., Conner, M., Kellar, I., Benn, Y., \& Sheeran, P. (2015). Does Monitoring Goal Progress Promote Goal Attainment? A Meta-Analysis of the Experimental Evidence. Psychological Bulletin, in Press. http://dx.doi.org/10.1037/bul0000025

Herman, C. W., Musich, S., Lu, C., Sill, S., Young, J. M., \& Edington, D. W. (2006). Effectiveness of an Incentive Based Online Physical Activity Intervention on Employee Health Status. Journal of Occupational and Environmental Medicine, 48, 889-895. http://dx.doi.org/10.1097/01.jom.0000232526.27103.71

Higaki, Y. (2013). Effects of Mild-Intensity Exercise on Metabolic Syndrome. Japanese Journal of Psychosomatic Medicine, 53, 237-246. (In Japanese)

Kessler, R. C., Andrews, G., Colpe, L. J., Hiripi, E., Mroczek, D. K., Normand, S. L., Walters, E. E., \& Zaslavsky, A. M. (2002). Short Screening Scales to Monitor Population Prevalences and Trends in Non-Specific Psychological Distress. Psychological Medicine, 32, 959-976. http://dx.doi.org/10.1017/S0033291702006074

Kessler, R. C., Barber, C., Beck, A., Berglund, P., Cleary, P. D., McKenas, D., Pronk, N., Simon, G., Stang, P., Üstün, T. U., \& Wang, P. (2003). The World Health Organization Health and Work Performance Questionnaire (HPQ). Journal of Occupational and Environmental Medicine, 45, 156-174. http://dx.doi.org/10.1097/01.jom.0000052967.43131.51

Kilkkinen, A., Kao-Philpot, A., O’Neil, A., Philpot, B., Reddy, P., Bunker, S., \& Dunbar, J. (2007). Prevalence of Psychological Distress, Anxiety and Depression in Rural Communities in Australia. The Australian Journal of Rural Health, 15, 114-119. http://dx.doi.org/10.1111/j.1440-1584.2007.00863.x

Kuroda, S. (2010). Do Japanese Work Shorter Hours than Before? Measuring Trends in Market Work and Leisure Using 1976-2006 Japanese Time-Use Survey. Journal of the Japanese and International Economies, 24, 481-502.

http://dx.doi.org/10.1016/j.jjie.2010.05.001

Kuroda, S. (2013). Current Situation regarding a Way of Working and Working Hours in Japan. Cabinet Office, Government of Japan, the Material of Council for Regulatory Reform. (In Japanese) http://www8.cao.go.jp/kisei-kaikaku/kaigi/meeting/2013/wg2/koyo/131031/item2.pdf

Merrill, R. M., Aldana, S. G., Garrett, J., \& Ross, C. (2011). Effectiveness of a Workplace Wellness Program for Maintaining Health and Promoting Healthy Behaviors. Journal of Occupational and Environmental Medicine, 53, $782-787$. http://dx.doi.org/10.1097/JOM.0b013e318220c2f4

Ministry of Health, Labour, and Welfare (2013). Occupational Safety and Health, Special Investigation (Laborer Health Survey). (In Japanese) http://www.mhlw.go.jp/toukei/list/dl/h24-46-50_01.pdf

Mimura, C., \& Griffiths, P. (2007). A Japanese Version of the Rosenberg Self-Esteem Scale: Translation and Equivalence Assessment. Journal of Psychosomatic Research, 62, 589-594. http://dx.doi.org/10.1016/j.jpsychores.2006.11.004

Mori, M., Tajima, M., Kimura, R., Sasaki, N., Somemura, H., Ito, Y. et al. (2014). A Web-Based Training Program Using Cognitive Behavioral Therapy to Alleviate Psychological Distress among Employees: Randomized Controlled Pilot Trial. JMIR Research Protocols, 3, e70. http://dx.doi.org/10.2196/resprot.3629

Murase, N., Katsumura, T., Ueda, C., Inoue, S., \& Shimomitsu, T. (2002). Validity and Reliability of Japanese Version of International Physical Activity Questionnaire. Journal of Health and Welfare Statistics, 49, 1-9. (In Japanese)

Ono, Y., \& Yoshimura, K. (2008). Japanese Version of WHO SUBI. Kaneko Shobo.

Pratt, L. A. (2009). Serious Psychological Distress, as Measured by the K6, and Mortality. Annals of Epidemiology, 19, $202-$ 209. http://dx.doi.org/10.1016/j.annepidem.2008.12.005

Proper, K. I., Koning, M., van der Beek, A. J., Hildebrandt, V. H., Bosscher, R. J., \& van Mechelen, W. (2003). The Effectiveness of Worksite Physical Activity Programs on Physical Activity, Physical Fitness, and Health. Clinical Journal of Sport Medicine, 13, 106-117. http://dx.doi.org/10.1097/00042752-200303000-00008

Robroek, S. J., van Lenthe, F. J., van Empelen, P., \& Burdorf, A. (2009). Determinants of Participation in Worksite Health Promotion Programmes: A Systematic Review. International Journal of Behavioral Nutrition and Physical Activity, 6, 26. http://dx.doi.org/10.1186/1479-5868-6-26

Rosenberg, M. (1965). Society and the Adolescent Self-Image. Princeton, NJ: Princeton University Press.

Sell, H., \& Nagpal, R. (1992). Assessment of Subjective Well-Being. The Subjective Well-Being Inventory (SUBI). Regional Health Paper, SEARO, 24, New Delhi: Regional Office for South-East Asia, World Health Organization. 
Tanaka, H. (2015). Beneficial Effect of Aerobic Exercise with Smile for Prevention and Treatment of obesity. Himan Kenkyu, 21, 94-98. (In Japanese)

Tsiros, M. D., Sinn, N., Brennan, L., Coates, A. M., Walkley, J. W., Petkov, J., Howe, P. R., \& Buckley, J. D. (2008). Cognitive Behavioral Therapy Improves Diet and Body Composition in Overweight and Obese Adolescents. American Journal of Clinical Nutrition, 87, 1134-1140.

Wolever, R. Q., Bobinet, K. J., McCabe, K., Mackenzie, E. R., Fekete, E., Kusnick, C. A., \& Baime, M. (2012). Effective and Viable Mind-Body Stress Reduction in the Workplace: A Randomized Controlled Trial. Journal of Occupational Health Psychology, 17, 246-258. http://dx.doi.org/10.1037/a0027278

Weissman, J. F., Pratt, L. A., Miller, E. A., \& Parker, J. D. (2015). Serious Psychological Distress among Adults: United States, 2009-2013. NCHS Data Brief, 203, 1-8.

Yerkes, R. M., \& Dodson, J. D. (1908). The Relation of Strength of Stimulus to Rapidity of Habit-Formation. Journal of Comparative Neurology and Psychology, 18, 459-482. http://dx.doi.org/10.1002/cne.920180503

Yoshimura, K., Kawakami, N., Tsutsumi, A., Inoue, A., Kobayashi, Y., Takenouchi, A., \& Fukuda, K. (2013). Cost-Benefit Analysis of Primary Prevention Programs for Mental Health at the Workplace in Japan. Occupational Health Journal, 55, 11-24. (In Japanese) http://dx.doi.org/10.1539/sangyoeisei.e12003 